\title{
HEALTH EFFECTS CLASSIFICATION AND ITS ROLE IN THE DERIVATION OF MINIMAL RISK LEVELS: NEUROLOGICAL EFFECTS
}

\author{
C-H SELENE JEN CHOU AND MILDRED WILLIAMS-JOHNSON
}

\author{
Agency for Toxic Substances and Disease Registry \\ Public Health Service \\ Department of Health and Human Services \\ Atlanta, Georgia
}

\begin{abstract}
The Agency for Toxic Substances and Disease Registry (ATSDR) uses substance-specific minimal risk levels (MRLs) to assist in evaluating public health risks associated with exposure to hazardous substances. By definition, "MRLs are estimates of daily human exposure to a chemical that are likely to be without an appreciable risk of adverse noncancer health effects over a specified duration of exposure." MRLs serve as screening levels for health assessors to identify contaminants and potential health effects that may be of concern for population living near hazardous waste sites and chemical releases. MRLs for each substance are derived for acute (1-14 days), intermediate (15-364 days), and chronic (365 days and longer) exposure durations, and for the oral and inhalation routes of exposure. The MRLs are derived from data compiled from a current comprehensive literature search and are presented in ATSDR's toxicological profile for that substance. In this paper we outline ATSDR's guidance for evaluating the neurological end point as discussed in the agency's toxicological profiles. Ranking neurological effects into less serious and serious categories and applying this procedure to the derivation of health guidance values or MRLs are also described. Specific examples of ATSDR MRLs based on neurological effects are presented.
\end{abstract}

\section{INTRODUCTION}

To determine the levels of significant human exposure to a given chemical associated with health effects, the Agency for Toxic Substances and Disease Registry (ATSDR) examines and interprets available toxicological and epidemiological data. The reported health effects are categorized according to severity: the no-observed-adverse-effect level (NOAEL), the less serious lowestobserved-adverse-effect level (LOAEL), and the serious LOAEL. In its Guidance for Developing Toxicological Profiles, ATSDR defines an adverse effect as "any effect that enhances the

1. Address all correspondence to: Selene Chou, Ph.D., Agency for Toxic Substances and Disease Registry, 1600 Clifton Road, MS E-29, N.E., Atlanta, GA 30333. Tel::(404)639-5287. Fax:(404)639-6315. E-mail:cjc3@cdc.gov.

2. Abbreviations: ATSDR, Agency for Toxic Substances and Disease Registry; LOAEL, lowest-observed-adverse-effect level; MF, modifying factor; MRLs, minimal risk levels; NOAEL, noobserved-adverse-effect-level; UF, uncertainty factor.

3. Key words: health guidance values, neurological effects, non-cancer risk assessment.

Toxicology and Industrial Health, Vol. 14, No. 3, pp. 455-471

Copyright $\odot 1998$ Princeton Scientific Publishing Co., Inc.

ISSN: 0748-2337 
susceptibility of an organism to the deleterious effects of other chemical, physical, microbiological, or environmental influences" (ATSDR, 1994). A dose that evokes failure in a biological system and can lead to morbidity or mortality is referred to as a serious LOAEL. After having compiled and evaluated the current database of toxicological and epidemiological studies, ATSDR derives minimal risk levels (MRLs) for the profiled substances. An MRL is an estimate of the daily human exposure to a chemical that is likely to be without appreciable risk of adverse noncancer health effects over a specified duration of exposure. MRLs are derived using the NOAEL/ uncertainty factor approach. Though there are inherent uncertainties with this method, these chemical-specific estimates are intended to serve as screening levels for health assessors to identify contaminants and potential health effects that may be of concern for populations living near hazardous waste sites and chemical releases. They are not intended to define clean-up or action levels.

\section{METHODS}

Derivation of Minimal Risk Levels

MRLs are derived using the NOAEL/uncertainty factor approach and are based on the highest NOAEL or lowest LOAEL reported in the substance-specific database. They are derived for acute (1-14 days), intermediate (15-364 days), and chronic (365 days and longer) exposure durations, and for the oral and inhalation routes of exposure. Neither cancer nor serious health effects are used as the basis for deriving MRLs. Thus, MRLs are based on non-neoplastic health end points and cancer effects are not a consideration (ATSDR, 1996; Chou et al., 1998). MRLs are derived based on the highest NOAEL not exceeding a LOAEL, or in the absence of a NOAEL, the lowest less serious LOAEL for the most sensitive health effect endpoint for a given route and exposure period in the database. An uncertainty factor (UF) is used to account for extrapolation from a LOAEL to a NOAEL. Additional UFs may also be used for human variability, for interspecies extrapolation when animal studies are used in the absence of adequate human data, and for extrapolation across exposure durations. In addition, a modifying factor (MF) may be used, on a case-by-case basis, to reflect concerns about the database not covered by the UFs. Thus,

$$
\mathrm{MRL}=\mathrm{NOAEL}(\text { or LOAEL) } /(\text { product of UFs } \times \text { MF) }
$$

MRLs for each substance are derived from data compiled from a current worldwide literature search and are presented in ATSDR's toxicological profiles for that substance. Proposed MRLs undergo review by a Health Effects/MRL Workgroup within the Division of Toxicology, external expert peer reviewers, and an agency-wide MRL Workgroup (with participation from other federal agencies), and are submitted for public comment through the toxicological profile public comment process. Each MRL is subject to change as new information becomes available concomitant with updating the toxicological profile for the substance. 
TABLE 1. Neurological Effects ${ }^{\mathrm{a}}$

\begin{tabular}{|c|c|c|}
\hline EFFECT & Less serious & Serious \\
\hline \multicolumn{3}{|l|}{ MOTOR } \\
\hline \multicolumn{3}{|l|}{$\begin{array}{l}\text { Activity changes (sedation, anesthesia, } \\
\text { somnolence, hyperactivity/hypoactivity, }\end{array}$} \\
\hline$\downarrow \quad$ locomotor activity) & $++^{\mathrm{b}}$ & + \\
\hline Convulsions & & + \\
\hline \multicolumn{3}{|l|}{$\begin{array}{l}\text { Lack of coordination (unsteadiness, intoxication, } \\
\downarrow \quad \text { swimming response ability, }\end{array}$} \\
\hline$\downarrow \quad$ psychomotor performance, ataxia) & $++^{b}$ & + \\
\hline Paralysis & & + \\
\hline Reflex abnormalities & $t^{b}$ & + \\
\hline Tremor, twitching (muscular spasm) & & + \\
\hline Weakness & + & \\
\hline \multicolumn{3}{|l|}{ MOOD AND PERSONALITY } \\
\hline Excitability & + & \\
\hline Delirium & & + \\
\hline Depression & $t^{b}$ & + \\
\hline Hallucinations & & + \\
\hline Irritability & + & \\
\hline Nervousness, tension & + & \\
\hline Restlessness & + & \\
\hline Sleep disturbances & + & \\
\hline \multicolumn{3}{|l|}{ SENSORY } \\
\hline Auditory disorders & & + \\
\hline Equilibrium changes & $t^{b}$ & + \\
\hline Pain disorders & $t^{b}$ & + \\
\hline Tactile disorders & $\dot{t}^{\mathbf{b}}$ & + \\
\hline Vision disorders & & + \\
\hline \multicolumn{3}{|l|}{ COGNITIVE } \\
\hline Confusion & & + \\
\hline Learning impairment ( $\downarrow$ operant behavior) & $t^{\mathrm{b}}$ & + \\
\hline Memory problems & & + \\
\hline Speech impairment & & + \\
\hline \multicolumn{3}{|l|}{ GENERAL } \\
\hline Depression of neuronal activity & $+b$ & + \\
\hline Fatigue (lethargy) & + & \\
\hline Loss of appetite & + & \\
\hline Narcosis, stupor & & + \\
\hline Nerve damage & & + \\
\hline Prostration & & + \\
\hline Other integrative effects (hand/eye coordination) & $t^{b}$ & + \\
\hline Unconsciousness & & + \\
\hline
\end{tabular}


TABLE 1. Neurological Effects ${ }^{\mathrm{a}}$ (cont'd)

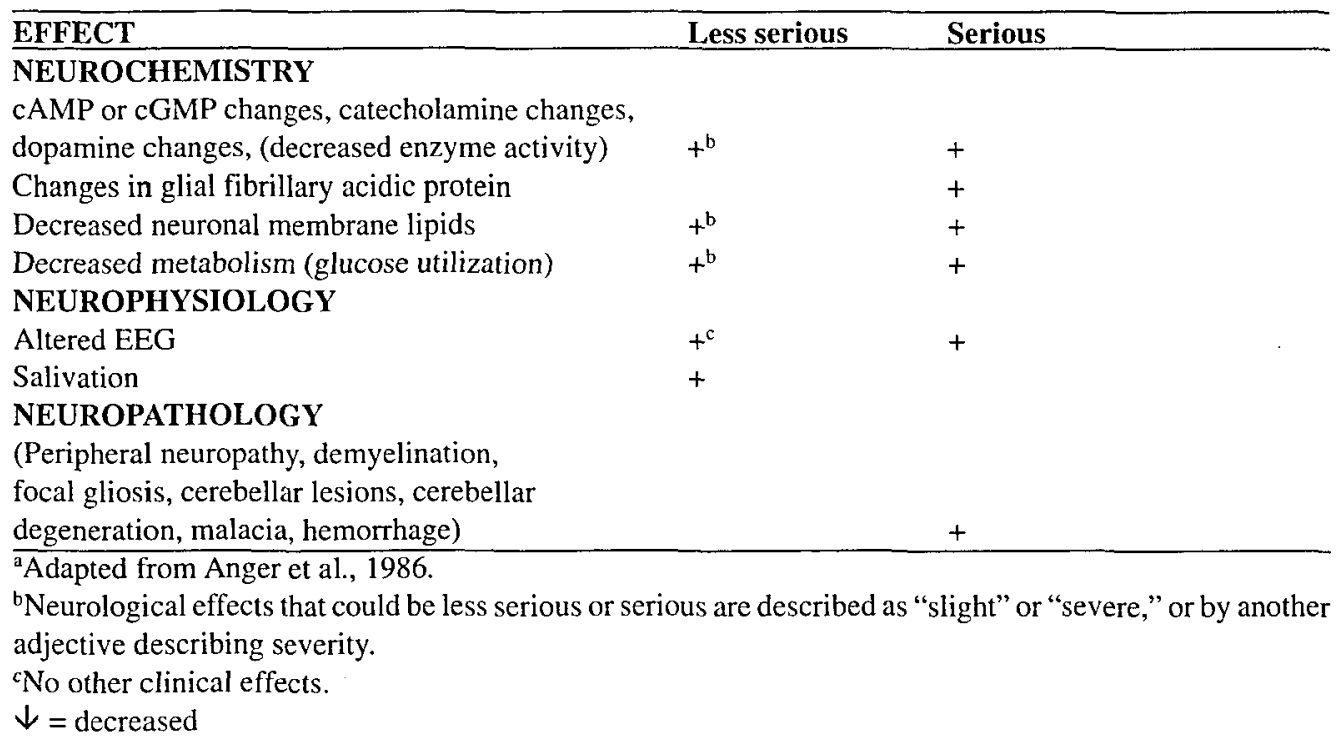

\section{Assessment of Neurological Effects}

In the ATSDR guidance for the preparation of a ninth set toxicological profile (ATSDR, 1995), neurotoxicity is defined as any adverse effect on the structure or function of the central or peripheral nervous system by a biological, chemical, or physical agent. Neurological effects may be permanent or reversible, produced by neuropharmacological or neurodegenerative properties of a neurotoxicant, or may be the result of direct or indirect actions on the nervous system. Neurological effects can be categorized as motor, mood and personality, sensory, cognitive, neurochemical, neurophysiologic, or neuropathologic. To provide guidance to agency scientists, specific end points within these effect categories have been listed and classified as less serious or serious (Table 1). This listing is not intended to be inclusive of all possible neurological changes that may have been reported.

\section{Cholinesterase Activity Inhibition}

Inhibition of acetylcholinesterase results in accumulation of acetylcholine at synapses and neuromuscular junctions. Exposure to pesticides such as organophosphorus compounds may produce a broad spectrum of clinical symptoms such as headaches, weakness, dizziness, blurred vision, psychosis, respiratory difficulty, paralysis, convulsions, and coma. Kaloyanova and El Batawi (1991) have reported correlation of inhibition of erythrocyte/brain cholinesterase activity with clinical symptoms (Table 2). 
TABLE 2. Acetylcholinesterase Activity (AChEA) Inhibition versus Severity of Neurological Symptoms $s^{a}$

\begin{tabular}{ll}
\hline Level of AChEA Inhibition & Severity of Neurological Symptoms \\
\hline$<60 \%$ reduction of AChEA & Mild \\
$60-90 \%$ reduction of AChEA & Moderate \\
$90-100 \%$ reduction of AChEA & Severe \\
\hline
\end{tabular}

${ }^{\mathrm{a} A d a p t e d ~ f r o m ~ K a l o y a n o v a ~ a n d ~ E l ~ B a t a w i, ~} 1991$.

In classifying the neurological health effect end point for inhibition of erythrocyte and/or brain acetylcholinesterase activity, ATSDR considers an exposure level that causes a 20-59\% inhibition of enzyme activity a less serious LOAEL, and an exposure level that causes $60 \%$ or greater inhibition of enzyme activity a serious LOAEL. In addition, considerations are given to associated clinical symptoms. If clinical effects observed at a particular exposure level are most consistent with a moderate or severe classification, this exposure level is classified as a serious LOAEL, even if the degree of inhibition of acetylcholinesterase activity is less than $60 \%$. Inhibition of acetylcholinesterase activity of $60 \%$ or greater is always classified as a serious effect.

\section{RESULTS AND DISCUSSION}

As mentioned previously, MRLs are based on the most sensitive end point. End points that may be used for MRL derivation include systemic effects (respiratory effects, cardiovascular effects, gastrointestinal effects, hematological effects, musculoskeletal effects, hepatic effects, renal effects, endocrine effects, dermal effects, ocular effects, body weight effects, metabolic effects), immunological and lymphoreticular effects, neurological effects, reproductive effects, and developmental effects. As of October 1997, ATSDR has derived 273 MRLs for 134 profiled substances. The neurological effects are among the most frequently used end points for deriving MRLs. To date 70 MRLs are based on neurological effects. Pertinent information on these MRLs and the associated health effects are shown in Table 3. Forty-four of the MRLs were based on neurological effects reported in animal studies, most of which were studies in rats (28 total rat studies). Studies in mice, dogs, rabbits, and gerbils were also used as the basis for deriving MRLs. NOAELs for neurological effects were reported in 38 of these investigations; MRL guidance values were derived from NOAELs reported for neurological effects in 31 of 44 studies in animals.

Among the neurological categories, 30 MRLs were derived from effects on motor function: 11 in humans and 19 in animals. Inhibition of acetylcholinesterase accounted for 15 MRLs: 1 in humans and 14 in animals. It should be noted that 14 MRLs based on developmental neurotoxicity were considered to be based on "developmental effects" and are therefore not included in Table 3. 
TABLE 3. MRLs Based on Neurological Effects. As of October 1997

\begin{tabular}{|c|c|c|c|c|c|c|}
\hline $\begin{array}{l}\text { Substance Name } \\
\text { (Tox profile status \& cover date) }\end{array}$ & Route & Duratio & MRL value & UFs $\times$ MF & Health Effects & Principal Study \\
\hline Acetone (Final 5/94) & Inhal & Acute & $26 \mathrm{ppm}$ & 9 & $\begin{array}{l}\text { LOAEL in humans; } \\
\text { increases in response and } \\
\% \text { false negatives in auditory } \\
\text { discrimination; increased anger, } \\
\text { hostility. }\end{array}$ & Dick et al., 1989 \\
\hline Acetone (Final 5/94) & Inhal & $\begin{array}{l}\text { Interm } \\
\& \text { chroni }\end{array}$ & $13 \mathrm{ppm}$ & 100 & $\begin{array}{l}\text { LOAEL in humans; } \\
\text { increased visual evoked response. }\end{array}$ & Stewart et al., 1975 \\
\hline Acrylonitrile (Final 12/90) & Inhal & Acute & $0.1 \mathrm{ppm}$ & 10 & NOAEL in humans. & Jakubowski et al., 1987 \\
\hline Benzene (Final 9/97) & Inhal & Interm & $0.004 \mathrm{ppm}$ & 90 & $\begin{array}{l}\text { LOAEL in mice; increased rapid } \\
\text { response time. }\end{array}$ & Li et al., 1992 \\
\hline Bromoform (Final 12/90) & Oral & Acute & $0.6 \mathrm{mg} / \mathrm{kg} / \mathrm{d}$ & 100 & LOAEL in humans; sedation. & Dwelle, 1903 \\
\hline Bromomethane (Final 9/92) & Inhal & Acute & $0.05 \mathrm{ppm}$ & 100 & $\begin{array}{l}\text { NOAEL in rats; decreased brain } \\
\text { neurotransmitters at higher dose. }\end{array}$ & Honma, 1987 \\
\hline Bromomethane (Final 9/92) & Inhal & Interm & $0.05 \mathrm{ppm}$ & 100 & $\begin{array}{l}\text { NOAEL in rats; decreased brain } \\
\text { neurotransmitters at higher dose. }\end{array}$ & Honma et al., 1982 \\
\hline Bromomethane (Final 9/92) & Inhal & Chronic & $0.005 \mathrm{ppm}$ & 100 & $\begin{array}{l}\text { LOAEL in humans; increased } \\
\text { prevalence of muscle ache, fatigue, } \\
\text { and ataxia. }\end{array}$ & Anger et al., 1986 \\
\hline Carbon disulfide (Final 8/96) & Inhal & Chronic & $0.3 \mathrm{ppm}$ & 30 & $\begin{array}{l}\text { LOAEL in humans; decreased } \\
\text { peroneal motor nerve conduction } \\
\text { velocity (MCV) and sural nerve } \\
\text { sensory conduction velocity (SCV) }\end{array}$ & Johnson et al., 1983 \\
\hline Chlorfenvinphos (Final 9/97) Oral & Acute & $0.002 \mathrm{mg}$ & $/ \mathrm{kg} / \mathrm{day}$ & 1000 & $\begin{array}{l}\text { LOAEL in rats; inhibition of plasma } \\
\text { and erythrocyte cholinesterase activity. }\end{array}$ & Barna and Simon, 1973 \\
\hline Chlorfenvinphos (Final 9/97) Oral & Chronic & $0.0007 \mathrm{~m}$ & $\mathrm{~g} / \mathrm{kg} / \mathrm{day}$ & 1000 & $\begin{array}{l}\text { LOAEL in rats; inhibition of plasma } \\
\text { and erythrocyte cholinesterase activity. }\end{array}$ & Ambrose et al., 1970 \\
\hline Chloroethane (Final 12/89) Inhal & Acute & $1300 \mathrm{ppn}$ & & 10 & $\begin{array}{l}\text { NOAEL in humans; intoxication } \\
\text { at higher dose. }\end{array}$ & Davidson. 1925 \\
\hline Chloromethane (Draft 9/97) Inhal & Acute & $0.5 \mathrm{ppm}$ & & 100 & $\begin{array}{l}\text { NOAEL in mice; cerebellar granule } \\
\text { cell degeneration at higher dose. }\end{array}$ & Landry et al., 1985 \\
\hline
\end{tabular}


TABLE 3. MRLs Based on Neurological Effects. As of October 1997 (cont'd)

\begin{tabular}{|c|c|c|c|c|c|c|}
\hline Substance Name & Route & Duration & MRL value UFs & $\times \mathbf{M F}$ & Health Effects & Principal Study \\
\hline Chloromethane (Draft 9/97) & Inhal & Chronic & $0.05 \mathrm{ppm}$ & 1000 & $\begin{array}{l}\text { LOAEL in mice; axonal swelling } \\
\text { and slight degeneration of axons } \\
\text { in spinal cord. }\end{array}$ & CIIT, 1981 \\
\hline Chlorpyrifos (Final 9/97) & Oral & Acute & $0.003 \mathrm{mg} / \mathrm{kg} / \mathrm{day}$ & 10 & $\begin{array}{l}\text { NOAEL in humans; runny nose, } \\
\text { blurred vision at higher dose. }\end{array}$ & Coulston et al., 1972 \\
\hline Chlorpyrifos (Final 9/97) & Oral & Interm & $0.003 \mathrm{mg} / \mathrm{kg} / \mathrm{day}$ & 10 & $\begin{array}{l}\text { NOAEL in humans; runny nose, } \\
\text { blurred vision at higher dose. }\end{array}$ & Coulston et al., 1972 \\
\hline Chlorpyrifos (Final 9/97) & Oral & Chronic & $0.001 \mathrm{mg} / \mathrm{kg} / \mathrm{day}$ & 100 & $\begin{array}{l}\text { NOAEL in rats; decreased red } \\
\text { blood cell ChE activity at higher dose. }\end{array}$ & McCollister et al., 1974 \\
\hline Cresol, ortho- (Final 7/92) & Oral & Acute & $0.05 \mathrm{mg} / \mathrm{kg} /$ day & 100 & $\begin{array}{l}\text { NOAEL in rabbits; hypoactivity at } \\
\text { higher dose. }\end{array}$ & BRRC, 1988 \\
\hline Cresol, para- (Final 7/92) & Oral & Acute & $0.05 \mathrm{mg} / \mathrm{kg} /$ day & 100 & $\begin{array}{l}\text { NOAEL in rabbits; hypoactivity at } \\
\text { higher dose. }\end{array}$ & BRRC, 1988 \\
\hline HMX (Final 9/97) & Oral & Acute & $0.1 \mathrm{mg} / \mathrm{kg} / \mathrm{day}$ & 1000 & $\begin{array}{l}\text { LOAEL in mice; } \\
\text { hyperkinesia when aroused } \\
\text { at higher dose. }\end{array}$ & Army, 1985 \\
\hline RDX (Final 6/95) & Oral & Acute & $0.06 \mathrm{mg} / \mathrm{kg} /$ day & 100 & $\begin{array}{l}\text { NOAEL in rats; } \\
\text { Convulsion, prostration } \\
\text { in dams at higher dose. }\end{array}$ & Army, 1986 \\
\hline Diazinon (Final 8/96) & Inhal & Interm & $0.009 \mathrm{mg} / \mathrm{m}^{3}$ & 30 & $\begin{array}{l}\text { NOAEL in rats; inhibition of } \\
\text { brain cholineesterase at higher dose }\end{array}$ & Hartmann, 1990 \\
\hline Diazinon (Final 8/96) & Oral & Interm & $0.0002 \mathrm{mg} / \mathrm{kg} / \mathrm{day}$ & 100 & $\begin{array}{l}\text { NOAEL in dogs; inhibition } \\
\text { of erythrocyte and brain } \\
\text { acetylcholine esterase, and } \\
\text { emesis at higher dose. }\end{array}$ & Barnes, 1988 \\
\hline Dichlorvos (Final 9/97) & Inhal & Acute & $0.002 \mathrm{ppm}$ & 100 & $\begin{array}{l}\text { NOAEL in rats; inhibition of } \\
\text { erythrocyte- acetylcholine esterase } \\
\text { at higher dose. }\end{array}$ & Schmidt et al., 1979 \\
\hline Dichlorvos (Final 9/97) & Inhal & Interm & $0.0003 \mathrm{ppm}$ & 100 & $\begin{array}{l}\text { NOAEL in rats; inhibition of } \\
\text { erythrocyte and brain } \\
\text { acetylcholine- esterase } \\
\text { at higher doses. }\end{array}$ & Thorpe et al., 1972 \\
\hline
\end{tabular}


TABLE 3. MRLs Based on Neurological Effects. As of October 1997 (cont'd)

\begin{tabular}{|c|c|c|c|c|c|c|}
\hline Substance Name & Route & Duration & n MRL value UFs & x MF & Health Effects & Principal Study \\
\hline CDichlorvos (Final 9/97) & Inhal & Chronic & $0.00006 \mathrm{ppm}$ & 100 & $\begin{array}{l}\text { NOAEL in rats; inhibition of } \\
\text { erythrocyte and brain } \\
\text { acetylcholine- esterase } \\
\text { at higher doses. }\end{array}$ & Blair et al., 1976 \\
\hline Dichlorvos (Final 9/97) & Oral & Acute & $0.004 \mathrm{mg} / \mathrm{kg} / \mathrm{day}$ & 1000 & $\begin{array}{l}\text { LOAEL in rats; inhibition of } \\
\text { brain acetylcholine- esterase. }\end{array}$ & Teichert et al., 1976 \\
\hline Dichlorvos (Final 9/97) & Oral & Interm & $0.003 \mathrm{mg} / \mathrm{kg} / \mathrm{day}$ & 10 & $\begin{array}{l}\text { NOAEL in humans for } \\
\text { erythrocyte acetylcholine-esterase } \\
\text { inhibition. }\end{array}$ & Boyer et al., 1977 \\
\hline Dichlorvos (Final 9/97) & Oral & Chronic & $0.0005 \mathrm{mg} / \mathrm{kg} / \mathrm{day}$ & 100 & $\begin{array}{l}\text { NOAEL in dogs; inhibition } \\
\text { of erythrocyte and brain } \\
\text { acetylcholine- esterase } \\
\text { at higher doses. }\end{array}$ & $\begin{array}{l}\text { AMVAC } \\
\text { Chemical Corp., } 1990\end{array}$ \\
\hline Disulfoton (Final 8/95) & Inhal & Acute & $0.006 \mathrm{mg} / \mathrm{m}^{3}$ & 30 & $\begin{array}{l}\text { NOAEL in rats; inhibition of } \\
\text { erythrocyte choline-esterase } \\
\text { and unspecified behavioral } \\
\text { disorders at higher doses. }\end{array}$ & Thyssen, 1978 \\
\hline Disulfoton (Final 8/95) & Inhal & Interm & $0.0002 \mathrm{mg} / \mathrm{m}^{3}$ & 30 & $\begin{array}{l}\text { NOAEL in rats; muscle tremors, } \\
\text { convulsions, increased salivation, } \\
\text { difficulty breathing at higher dose. }\end{array}$ & Thyssen, 1980 \\
\hline Disulfoton (Final 8/95) & Oral & Acute & $0.001 \mathrm{mg} / \mathrm{kg} / \mathrm{day}$ & 100 & $\begin{array}{l}\text { NOAEL in rats; inhibition of } \\
\text { plasma and erythrocyte cholinesterase } \\
\text { at higher doses. }\end{array}$ & Lamb and Hixon, 1983 \\
\hline Disulfoton (Final 8/95) & Oral & Chronic & $0.00006 \mathrm{mg} / \mathrm{kg} / \mathrm{day}$ & 1000 & $\begin{array}{l}\text { LOAEL in rats; } \\
\text { inhibition of erythrocyte and } \\
\text { brain cholinesterase. }\end{array}$ & Hayes, 1985 \\
\hline Endrin (Final 8/96) & Oral & Interm & $0.002 \mathrm{mg} / \mathrm{kg} / \mathrm{day}$ & 100 & $\begin{array}{l}\text { NOAEL in dogs; convulsions, } \\
\text { tremors, diffuse degenerative brain } \\
\text { lesions at higher dose. }\end{array}$ & Treon et al., 1955 \\
\hline
\end{tabular}


TABLE 3. MRLs Based on Neurological Effects. As of October 1997 (cont'd)

\begin{tabular}{|c|c|c|c|c|c|c|}
\hline Substance Name & Route & Duratio & aMRL value UFs & $\mathbf{x}$ MF & Health Effects & Principal Study \\
\hline Endrin (Final 8/96) & Oral & Chronic & $0.0003 \mathrm{mg} / \mathrm{kg} / \mathrm{day}$ & 100 & $\begin{array}{l}\text { NOAEL in dogs; } \\
\text { convulsions at higher dose. }\end{array}$ & Kettering, 1969 \\
\hline Fuel oil \#2 (Final 6/95) & Inhal & Acute & $0.02 \mathrm{mg} / \mathrm{m}^{3}$ & 1000 & $\begin{array}{l}\text { LOAEL in mice; ataxia, } \\
\text { disturbed gait. }\end{array}$ & Kainz and White, 1984 \\
\hline $\begin{array}{l}\text { Hexachlorocyclo-hexane, } \\
\text { gamma- (Draft 9/97) }\end{array}$ & Oral & Acute & $0.01 \mathrm{mg} / \mathrm{kg} /$ day & 100 & $\begin{array}{l}\text { NOAEL in rats; } \\
\text { increased kindling acquisition; } \\
\text { seizures at higher dose. }\end{array}$ & Joy, 1982 \\
\hline $\begin{array}{l}\text { Hexachlorocyclo-hexane, } \\
\text { beta- (Draft 9/97) }\end{array}$ & Oral & Acute & $0.2 \mathrm{mg} / \mathrm{kg} /$ day & 100 & $\begin{array}{l}\text { NOAEL in mice; } \\
\text { ataxia at higher dose. }\end{array}$ & Cornacoff et al., 1988 \\
\hline Hexachloro-ethane (Final 9/97) & Inhal & Acute & $6 \mathrm{ppm}$ & 30 & $\begin{array}{l}\text { NOAEL in rats; } \\
\text { tremors at higher dose. }\end{array}$ & Weeks et al., 1979 \\
\hline Hexachloro-ethane (Final 9/97) & Inhal & Interm & $6 \mathrm{ppm}$ & 30 & $\begin{array}{l}\text { NOAEL in rats; } \\
\text { tremors at higher dose. }\end{array}$ & Weeks et al., 1979 \\
\hline Chlordecone (Final 8/95) & Oral & Acute & $0.01 \mathrm{mg} / \mathrm{kg} /$ day & 100 & $\begin{array}{l}\text { NOAEL in rats; } \\
\text { increased startle response } \\
\text { at higher dose. }\end{array}$ & EPA, 1986 \\
\hline n-Hexane (Draft 9/97) & Inhal & Chronic & $0.6 \mathrm{ppm}$ & 100 & $\begin{array}{l}\text { LOAEL in humans; } \\
\text { decreased motor nerve } \\
\text { conduction velocity. }\end{array}$ & Sanagi et al., 1980 \\
\hline Manganese (Draft 10/97) & Oral & Chronic & $0.00004 \mathrm{mg} / \mathrm{m}^{3}$ & 900 & $\begin{array}{l}\text { LOAEL in humans; } \\
\text { decreased reaction time, } \\
\text { finger tapping. }\end{array}$ & Iregren, 1990 \\
\hline Mercury (Draft 8/97) & Inhal & Chronic & $0.0002 \mathrm{mg} / \mathrm{m}^{3}$ & 30 & $\begin{array}{l}\text { LOAEL in humans; } \\
\text { increased frequency of mild } \\
\text { intention tremors with weight load. }\end{array}$ & Fawer et al., 1983 \\
\hline Methyl parathion (Final 9/92)Oral & Chronic & $0.0003 n$ & $\mathrm{~g} / \mathrm{kg} / \mathrm{day}$ & 100 & $\begin{array}{l}\text { NOAEL in rats; abnormal gait, } \\
\text { slight tremors, peripheral neuropathy } \\
\text { and inhibition of cholinesterases } \\
\text { at higher doses. }\end{array}$ & Suba, 1984 \\
\hline
\end{tabular}

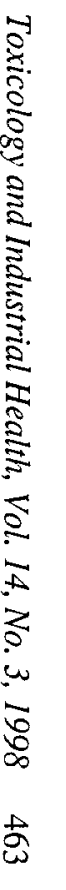


TABLE 3. MRLs Based on Neurological Effects. As of October 1997 (cont'd)

\begin{tabular}{|c|c|c|c|c|c|c|}
\hline $\begin{array}{l}\text { Substance Name } \\
\text { (Tox profile status \& cover date) }\end{array}$ & Route & Duration & MRL value $U$ & UFs $\times$ MF & Health Effects & Principal Study \\
\hline Methyl t-butyl ether (Final 8/96) & Inhal & Acute & $2 \mathrm{ppm}$ & 100 & $\begin{array}{l}\text { NOAEL in rats; } \\
\text { increased incidence/severity } \\
\text { of ataxia and duck walk gait. }\end{array}$ & Gill, 1989 \\
\hline Methyl t-butyl ether (Final 8/96) & Inhal & Interm & $0.7 \mathrm{ppm}$ & 100 & $\begin{array}{l}\text { NOAEL in rats; } \\
\text { hypoactivity, lack of startle } \\
\text { response, and blepharospasm } \\
\text { at higher dose. }\end{array}$ & Neeper-Bradley, 1991 \\
\hline Methyl t-butyl ether (Final 8/96) & Oral & Acute & $0.4 \mathrm{mg} / \mathrm{kg} / \mathrm{day}$ & 100 & $\begin{array}{l}\text { NOAEL in rats; } \\
\text { drowsiness at higher dose. }\end{array}$ & Bioresearch Labs, 1990 \\
\hline Methylene chloride (Final 4/93) & Inhal & Acute & $0.4 \mathrm{ppm}$ & 100 & $\begin{array}{l}\text { LOAEL in humans; critical } \\
\text { flicker frequency depression, } \\
\text { vigilance decrease; } \\
\text { impaired psychomotor tasks } \\
\text { at higher dose. }\end{array}$ & Winneke, 1974 \\
\hline Naphthalene (Final 8/95) & Oral & Acute & $0.05 \mathrm{mg} / \mathrm{kg} / \mathrm{day}$ & 1000 & $\begin{array}{l}\text { LOAEL in rats; lethargy, } \\
\text { slow breathing, increased rooting. }\end{array}$ & NTP, 1991 \\
\hline $\begin{array}{l}\text { Propylene Glycol Dinitrate } \\
\text { (Final 6/95) }\end{array}$ & Inhal & Acute & $0.003 \mathrm{ppm}$ & 10 & $\begin{array}{l}\text { NOAEL in humans; altered visual } \\
\text { evoked response, headache, and } \\
\text { ataxia at higher doses. }\end{array}$ & Stewart et al., 1974 \\
\hline Styrene (Final 9/92) & Inhal & Chronic & $0.06 \mathrm{ppm}$ & 100 & $\begin{array}{l}\text { LOAEL in humans; } \\
\text { decreased verbal learning skills. }\end{array}$ & Mutti et al., 1984 \\
\hline Tetrachloro-ethylene (Final 9/97) & Inhal & Acute & $0.2 \mathrm{ppm}$ & 10 & $\begin{array}{l}\text { NOAEL in humans; increased } \\
\text { latency of visual evoked potentials } \\
\text { at higher dose. }\end{array}$ & Altmann et al., 1990 \\
\hline Tetrachloro-ethylene (Final 9/97) & Inhal & Chronic & $0.04 \mathrm{ppm}$ & 100 & $\begin{array}{l}\text { LOAEL in humans; } \\
\text { increased reaction time. }\end{array}$ & Ferroni et al., 1992 \\
\hline Toluene (Final 5/94) & Inhal & Acute & $3 \mathrm{ppm}$ & 30 & $\begin{array}{l}\text { LOAEL in humans; } \\
\text { decreased manual dexterity } \\
\text { and visual perception. }\end{array}$ & Baelum et al., 1985 \\
\hline
\end{tabular}


TABLE 3. MRLs Based on Neurological Effects. As of October 1997 (cont'd)

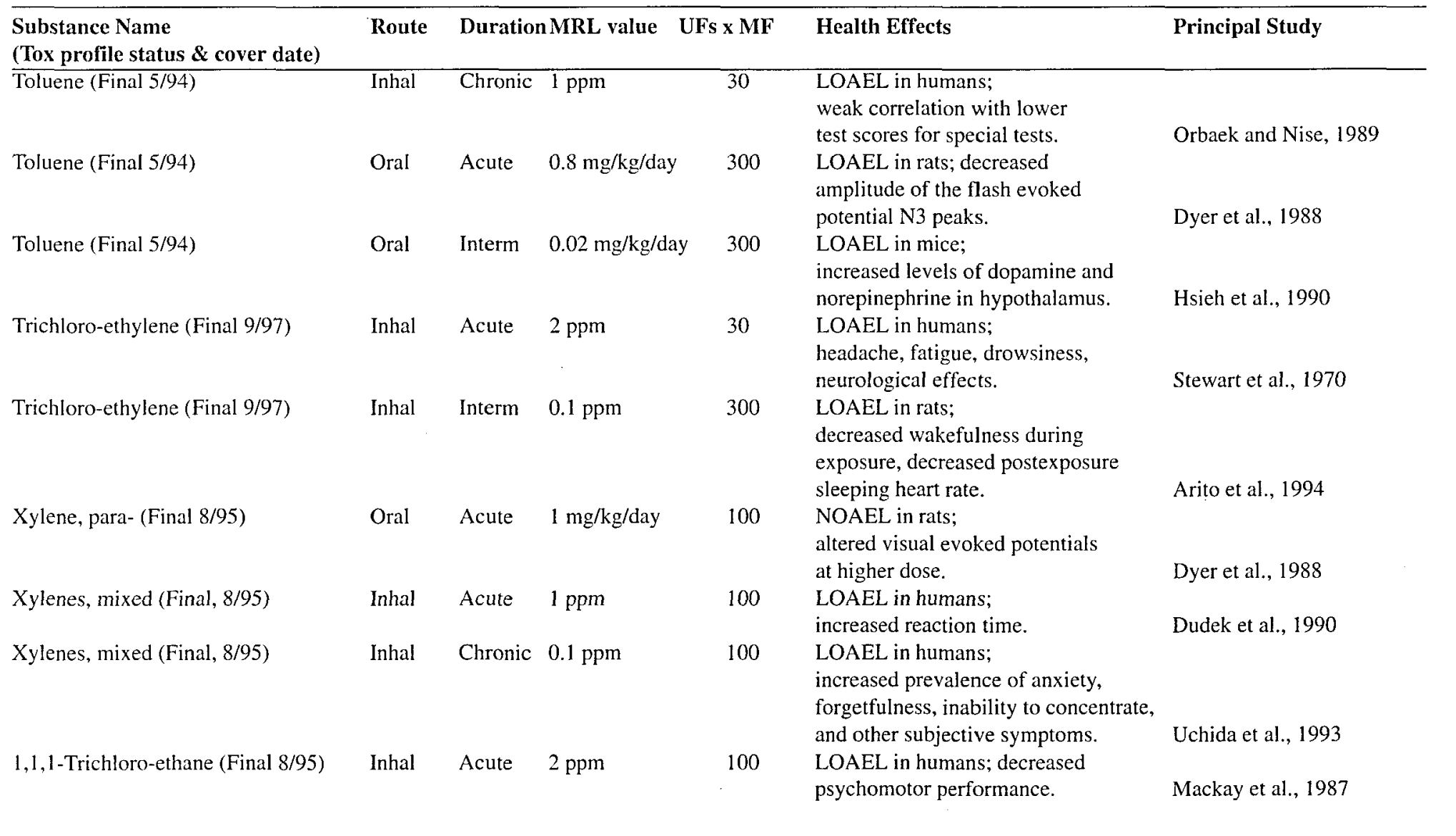


TABLE 3. MRLs Based on Neurological Effects. As of October 1997 (cont'd)

\begin{tabular}{|c|c|c|c|c|c|c|}
\hline \multirow{2}{*}{$\begin{array}{l}\text { Substance Name } \\
\text { (Tox profile status \& cover date) } \\
1,1,1 \text {-Trichloro- ethane (Final } 8 / 95)\end{array}$} & \multirow{2}{*}{$\frac{\text { Route }}{\text { Inhal }}$} & \multicolumn{2}{|c|}{ Duration MRL value } & UFs $\times$ MF & \multirow{2}{*}{$\begin{array}{l}\text { Health Effects } \\
\text { NOAEL in gerbils; } \\
\text { increased glial fibrillary acidic } \\
\text { protein indicating astrogliosis } \\
\text { at high dose. }\end{array}$} & \multirow{2}{*}{$\begin{array}{l}\text { Principal Study } \\
\\
\text { Rosengren et al. } 1985\end{array}$} \\
\hline & & Interm & $0.7 \mathrm{ppm}$ & 100 & & \\
\hline 1,1,2-Trichloro-ethane (Final 12/89) & Oral & Acute & $0.3 \mathrm{mg} / \mathrm{kg} /$ day & 100 & $\begin{array}{l}\text { NOAEL in mice; } \\
\text { taste aversion at higher dose. }\end{array}$ & Kallman et al., 1983 \\
\hline 1,2-Dichloro-propane (Final 12/89) & Oral & Acute & $0.1 \mathrm{mg} / \mathrm{kg} / \mathrm{day}$ & 1000 & LOAEL in rats; slight CNS depression. & Bruckner et al. 1989 \\
\hline 2,4-Dinitro-toluene (Draft 9/97) & Oral & Acute & $0.05 \mathrm{mg} / \mathrm{kg} /$ day & 100 & $\begin{array}{l}\text { NOAEL in dogs; incoordination, } \\
\text { stiffness, and abnormal gait } \\
\text { at higher dose. }\end{array}$ & $\begin{array}{l}\text { Ellis et al., 1985; } \\
\text { Lee et al., } 1978\end{array}$ \\
\hline 4,6-Dinitro-o-cresol (Final 8/95) & Oral & Acute & $0.004 \mathrm{mg} / \mathrm{kg} /$ day & 100 & $\begin{array}{l}\text { LOAEL in humans; } \\
\text { fatigue and dizziness. }\end{array}$ & Plotz, 1936 \\
\hline 4,6-Dinitro-o-cresol (Final 8/95) & Oral & Interm & $0.004 \mathrm{mg} / \mathrm{kg} /$ day & 100 & $\begin{array}{l}\text { LOAEL in humans; } \\
\text { fatigue and dizziness. }\end{array}$ & Plotz, 1936 \\
\hline
\end{tabular}


Classification of neurological effects into NOAEL, less serious LOAEL, or serious LOAEL is based on degree of severity. When a less serious LOAEL is used as the basis to derive an MRL, a default UF of 10 is generally used for extrapolation from a less serious LOAEL to a NOAEL. However, an effect level is considered to be a minimal LOAEL when only minimally toxic effects are observed that are thought to represent an early indication of toxicity. A UF of 3 is generally used to extrapolate from a minimal LOAEL to a NOAEL. Representative examples where a UF of 3 was used for use of a minimal LOAEL for neurological effects in deriving an MRL are as follows:

An acute inhalation MRL of $26 \mathrm{ppm}$ for acetone was derived based on mild decrements on behavioral performance tests in volunteers after a $4 \mathrm{~h}$. exposure to $237 \mathrm{ppm}$ acetone (Dick et al., 1989). There were small statistically significant changes in performance from controls in two measures of the auditory tone discrimination task, i.e., increased response time to correct hits and false alarm percent rate, and on the anger hostility scale (men only) of the profile of mood states test. In deriving the MRL, a UF of 9 was applied to the LOAEL of $237 \mathrm{ppm}, 3$ for use of a minimal LOAEL and 3 for human variability.

A chronic inhalation MRL of $0.3 \mathrm{ppm}$ for carbon disulfide was derived based on decreased peroneal motor nerve conduction velocity and sural nerve sensory conduction velocity in workers exposed occupationally to $7.6 \mathrm{ppm}$ for 12.1 years (S.D. 6.9 years) in comparison to controls. Although the small reductions in nerve conduction velocities were still within the range of clinically normal values, the authors concluded that this decrement should be considered a minimal neurotoxic effect (Johnson et al., 1983). In calculating the MRL, a UF of 30 (3 for use of a minimal LOAEL, and 10 for human variability) was applied to the LOAEL of $7.6 \mathrm{ppm}$.

An acute inhalation MRL of $3 \mathrm{ppm}$ for toluene was derived based on decreased psychomotor performance in manual dexterity and visual perception speed in workers exposed to $100 \mathrm{ppm}$ toluene for 6.5 hours in comparison to unexposed controls (Baelum et al., 1985). In deriving the MRL, a UF of 30 ( 3 for use of a minimal LOAEL, and 10 for human variability) was applied to the LOAEL of $100 \mathrm{ppm}$.

Because MRLs are derived through workgroup consensus, the scientific judgment of the collective body is employed. The underlying mechanism and significance of the observed neurological effects are not always clearcut. The expertise of the workgroup members, coupled with their ability to achieve consistency in making biomedical judgments across profiled substances is crucial in deriving health-based guidance values.

\section{ACKNOWLEDGMENTS}

The authors acknowledge the valuable contributions made by all MRL Workgroup members, past and present. We also thank Dr. Dennis Jones for providing critical reviews and comments, and Ms. Anne Olin for editing the manuscript. 


\section{REFERENCES}

ALTMANN, L., BOTTGER, A., and WIEGAND, H. (1990). "Neurophysiological and psychophysical measurements reveal effects of acute low-level organic solvent exposure in humans." Int. Arch. Occup. Environ. Health 62:493-499.

AMBROSE, A.M., LARSON, P.S., BORZELLECA, J.F., et al. (1970). "Toxicologic studies on diethyl-1(2,4-dichlorophenyl)-2-chlorovinyl phosphate." Toxicol. Appl. Pharmacol. 17:323-336.

AMVAC Chemical Corp. (1990). A 52-week Chronic Toxicity Study on DDVP in Dogs. Unpublished report dated August 6, 1990, submitted by AMVAC Chemical Corporation, Los Angeles, CA. EPA-41593101.

ANGER, W.K., MOODY, L., FURG, J., et al. (1986). "Neurobehavioral evaluation of soil and structural fumigators using methyl bromide and sulfuryl fluoride." Neurotoxicity 7:137-156.

ANGERHOFER et al. (1986). Teratological assessment of cyclotrimethylene trinitramine (RDX) in rats. Dept of the Army, U.S. Army Environmental Hygiene Agency. Document No. AD-A166 249. Aberdeen Proving Ground, MD.

ARITO, H., TAKAHASHI, M., and ISHIKAWA, T. (1994). "Effect of subchronic inhalation exposure to low-level trichloroethylene on heart rate and wakefulness-sleep in freely moving rats." Sangyo Igaku 36:1-8.

GREENOUGH, R.J. and MCDONALD, P. (1985). Cyclotetramethylene Tetranitramine (HMX): 14-day Toxicity Study in Mice by Dietary Administration. Research and Development Command, U.S. Army Medical Bioengineering Research and Development Laboratory. Ft. Detrick, MD.

Agency for Toxic Substances and Disease Registry (ATSDR) (1995). Guidance for the Preparation of a Toxicological Profiles.

Agency for Toxic Substances and Disease Registry (ATSDR) (1996). Minimal Risk Levels for Priority Substances and Guidance for Derivation. Federal Register 61:33511-33520.

BAELUM, J., ANDERSON, I., LUNDQVIST, G.R., et al. (1985). "Response of solvent-exposed printers and unexposed controls to six-hour toluene exposure." Scand. J. Work Environ. Health 59:281-294.

BARNA, J. and SIMON, G. (1973). "Effect of small oral doses of Birlane (chlorfenvinphos) on intestinal resorption." Kiserl Orvostud 26:606-609.

BARNES, T.B. (1988). 90-day Oral Toxicity Study in Dogs. Project No. 882012. Unpublished report dated August 4, 1988, from Ciba-Geigy Ltd. Basel, Switzerland. EPA-40815004.

BIORESEARCH LABORATORIES (1990). Mass Balance or Radioactivity and Metabolism in Male and Female Fischer-344 rats After Intravenous, Oral and Dermal Administration of ${ }^{14} \mathrm{C}$-Methyl Tertiary-Butyl Ether. Report No. 38843. Senneville, Quebec, Canada.

BLAIR, D., DIX, K.M., HUNT, P.F., et al. (1976). "Dichlorvos- a 2-year inhalation carcinogenesis study in rats." Arch. Toxicol. 35:281-294.

BOYER, A.C., BROWN, L.J., SLOMKA, M.B., et al. (1977). "Inhibition of human plasma cholinesterase by ingested dichlorvos: Effect of formulation vehicle.” J. Toxicol. Appl. Pharmacol. 41(2):389-394.

Bushy Run Research CenTer (BRRC) (1988). Developmental Toxicity Evaluation of o-, m-, or p-Cresol Administered by Gavage to New Zealand White Rabbits. Unpublished data submitted to EOA/OTS. Fiche No. OTS0517695.

BRUCKNER, J.V., MACKENZIE, W.F., RAMANATHAN, R., et al. (1989). "Oral toxicity of 1,2dichloropropane: Acute short-term and long-term studies in rats." Fund. Am. Appl. Toxicol. 12:713-730.

CHOU, C-H. S.J, HOLLER, J.S., and DE ROSA, C.T. (1998). “Minimal risk levels (MRLs) for hazardous substances." J. Clean Technol. Environ. Toxicol. Occup. Med. 7(1):1-24.

CIIT. (1981). Final Report on a Chronic Inhalation Toxicology Study in Rats and Mice Exposed to Methyl Chloride. OTS Submission Document ID 40-8120717. Microfiche 511310. Unpublished study prepared by Battelle-Columbus Laboratories, Columbus, $\mathrm{OH}$.

CORNACOFF, J.B., LAUER, L.D., HOUSE, R.V., et al. (1988). "Evaluation of the immunotoxicity of beta-hexachlorocyclohexane." Fund. Am. Appl. Toxicol. 11:293-299.

COULSTON, F., GOLDBERG, L., ABRAHAM, R., et al. (1972). Final Report on Safety Evaluation and Metabolic Studies on Dowco. 179(IN151). Institute of Experimental Pathology and Toxicology, Albany Medical College. Albany, NY. 
DAVIDSON, B.M. (1925). "Studies of intoxication V. The action of ethyl chloride." J. Pharmacol. Exper. Thera. 26:37-42.

DICK, R.B., SETZER, J.V., TAYLOR, B.J., et al. (1989). "Neurobehavioral effects of short duration exposures to acetone and methyl ethyl ketone." Br. J. Ind. Med. 46:111-121.

DUDEK, B., GRALEWICZ, K., JAKUBOWSKI, M., et al. (1990). "Neurobehavioral effects of experimental exposure to toluene, xylene and their mixture." Pol. J. Occup. Med. 3:109-116.

DYER, R.S., BERCEGEAY, M.S., and MAYO, L.M. (1988). "Acute exposures to p-xylene and toluene alter visual information processing." Neurotoxicol. Teratol. 10:147-153.

DWELLE, E.H. (1903). "Fatal bromoform poisoning." JAMA 41:1540.

ELLIS, H.V., HONG, C.B., LEE, C.C., et al. (1985). "Subchronic and chronic toxicity studies of 2,4dinitrotoluene. Part I. Beagle dog." J. Am. Coll. Toxicol. 4:233-242.

U.S. Environmental Protection Agency (USEPA) (1986). Final Report on the Evaluation of Four Toxic Chemicals in an "in vivo/in vitro" Toxicological Screen: Acrylamide, Chlordecone, Cyclophosphamide, and Diethylstilbestrol. U.S. Environmental Protection Agency, Health Effects Research Laboatory. EPA-600-1-86-002. Research Triangle Park, NC.

FAWER, R.F., DERIBAUPIERRE, Y., GUILLEMIN, M., et al. (1983). "Measurement of hand tremor induced by industrial exposure to metallic mercury." Br. J. Ind. Med. 40:204-208.

FERRONI, C., SELIS, L., MUTTI, A., et al. (1992). "Neurobehavioral and neuroendocrine effects of occupational exposure to perchloroethylene," Neurotoxicology 13:243-247.

GILL, M.W. (1989). Methyl Tertiary Butyl Ether Single Exposure Vapor Inhalation Neurotoxicity Study in Rats. Project Report 52-533. Bushy Run Research Center, Export, PA.

HARTMANN, H.R. (1990). 21-day repeated exposure inhalation toxicity in the rat, nose only exposure. Project No. 891205. EPA-41557402. Unpublished report dated June 8, 1990, from Ciba-Geigy Ltd,, Basel, Switzerland.

HAYES, R.H. (1985). Chronic Feeding/Oncogenicity Study of Technical Disulfoton (Di-Syston) with Rats. Study No. 82-271-01. Mobay Chemical Corporation, Kansas City, MO.

HONMA, T., SUDO, A., MIYAGAWA, M., et al. (1982). "Significant changes in monoamines in rat brain induced by exposure to methyl bromide." Neurobehav. Toxicol. Teratol. 4:521-524.

HONMA, T. (1987). "Alteration of catecholamine metabolism in rat brain produced by inhalation exposure to methyl bromide." Jpn. J. Ind. Health 29:218-219.

HSIEH, G.C., SHARMA, R.P., PARKER, R.D., et al.(1990). "Evaluation of toluene exposure via drinking water on levels of regional brain biogenic monoamines and their metabolites in CD-1 mice." Ecotoxicol. Environ. Safety 20:175-184.

IREGREN, A. (1990). "Psychological test performance in foundry workers exposed to low levels of manganese." Neurotoxicol. Teratol. 12:673-675.

JAKUBOWSKI, M., LINHART, I., PIELAS, G., et al. (1987). "2-Cyanoethylmercapturic acid in the urine as a possible indicator of exposure to acrylonitrile." Br. J. Ind. Med. 44:834-840.

JOHNSON, B.L., BOYD, J., BURG, J.R., et al. (1983). "Effects on the peripheral nervous system of worker's exposure to carbon disulfide." Neurotoxicology 4:53-65.

JOY, R. (1982). "Mode of action of lindane, dieldrin, and related insecticides in the central nervous system." Neurobehav. Toxicol. Teratol. 4:813-823.

KALOYANOVA, F.P. and EL BATAWI, M.A. (1991). Human Toxicology of Pesticides. CRC Press, Boca Raton, FL. pp. 3-57.

KAINZ, R.J. and WHITE, L.E. (1984). Depressant Effects Associated with the Inhalation of Uncombusted Diesel Vapor. In: Advances in Modern Environmental Toxicology. (H.N. MacFarland, C.E. Holdsworth, J.A. MacGregor, et al,, eds.) Volume VI: Applied toxicology of petroleum hydrocarbons. Princeton Scientific Publishers, Inc. Princeton, NJ.

KALLMAN, M.J., LYNCH, M.R., and LANDAUER, M.R. (1983). "Taste aversions to several halogenated hydrocarbons." Neurobehav. Toxicol. Teratol. 5:23-27.

Kettering Laboratory (1969). Effects Exerted Upon Beagle Dogs During a Period of Two Years by the Introduction of 1,2,3,4,10,10-hexachloro-6,7-expoxy-1,4,4a,5,6,7,8,8a-octahydro-1,4-endo,endo-5,8dimethanonaphthalene into Their Daily Diets. Report to Velsicol Chemical Corporation. Cincinnati, $\mathrm{OH}$. 
LAMB, D.W. and HIXON, E.J. (1983). Embryotoxic and Teratogenic Effects of Disulfoton. Study No. 81-611-02. Mobay Chemical Corporation, Kansas City, MO.

LANDRY, T.D., QUAST, J.F., and GUSHOW, T.S. (1985). "Neurotoxicity of methyl chloride in continuously versus intermittently exposed female C57BL/6 mice." Fund. Am. Appl. Toxicol. 5:87-98.

LEE, C.C., ELLIS, H.V., KOWALSKI, J.J., et al. (1978). Mammalian Toxicity of Munitions Compounds. Phase II; Effects of Multiple Doses. Part II: 2,4-Dinitrotoluene. Progress report No. 3. Contract No. DAMD 17-74-C-4073. Midwest Research Institute, Kansas City, MO.

LI, L., SUN, W., GONG, Z., et al. (1992). "Effect of low benzene exposure on neurobehavioral function, AChE in blood, brain and bone marrow picture in mice." Biomed. Environ. Sci. 5(4):349-354.

MACKAY, C.J., CAMPBELL, L., SAMUEL, A.M., et al. (1987). "Behavioral changes during exposure to 1,1,1-trichloroethane: time-course and relationship to blood solvent levels." Am. J. Ind. Med. 11:223-240.

MCCOLLISTER, S.B., KOCIBA, R.J., HUMISTON, C.G., et al. (1974). "Studies of the acute and long term oral toxicity of chlorpyrifos." Food Cosmet. Toxicol. 12(1):45-61.

MUTTI, A., MAZZUCCHI, A., RUSTICHELLI, P., et al. (1984). "Exposure-effect and exposure-response relationships between occupational exposure to styrene and neuropsychological functions." Am. J. Ind. Med. 5:275-286.

NEEPER-BRADLEY, T.L. (1991). Two-Generation Reproduction Study of Inhaled Methyl Tert-Butyl Ether in CD Sprague-Dawley Rats. Project ID 53-594. Bushy Run Research Center, Export, PA.

National Toxicology Program (NTP) (1991). Developmental Toxicity of Naphthalene Administered by Gavage to Sprague-Dawley (CD) Rats on Gestational Days 6 Through 15. National Toxicology Program, National Institute of Environmental Health Sciences, U.S. Department of Health and Human Services, Public Health Service, National Institutes of Health. TER-91006. Research Triangle Park, NC.

ORBAEK, P. and NISE, G. (1989). "Neurasthenic complaints and psychometric function of toluene-exposed rotogravure printers." Am. J. Ind. Med. 16:67-77.

PLOTZ, M. (1936). "Dinitro-ortho-cresol. A metabolic stimulator and its toxic side-actions." NY State J. Med. 41:266-268.

ROSENGREN, L.E., AURELL, A., KJELLSTRAND, P., et al. (1985). "Astrogliosis in the cerebral cortex of gerbils after long-term exposure to 1,1,1-trichlorocthane." Scand. J. Work Environ. Health $11: 447-456$.

SANAGI, S., SEKI, Y., SUGIMOTO, K., et al. (1980). "Peripheral nervous system functions of workers exposed to n-hexane at a low level." Int. Arch. Occup. Environ. Health 47(1):69-79.

SCHMIDT, G., SCHMIDT, M., NENNER, M., et al. (1979). "Effects of dichlorvos (DDVP) inhalation on the activity of acetylcholinesterase in the bronchial tissue of rats." Arch. Toxicol. 42(3):191-198.

STEWART, R.D., DODD, H.C., GAY, H.H., et al. (1970). "Experimental human exposure to trichloroethylene." Arch. Environ. Health 20:64-71.

STEWART, R.D., PETERSON, J.E., NEWTON, P.E., et al. (1974). "Experimental human exposure to propylene glycol dinitrate." Toxicol. Appl. Pharmacol. 30:377-395.

STEWART, R.D., HAKE, C.L., WU, A., et al. (1975). "Acetone: Development of a biologic standard for the industrial worker by breath analysis." National Institute for Occupational Safety and Health. NTIS PB82-172917. Cincinnati, OH.

SUBA, L.A. (1984). Additional Information to Support the Registration of Methyl Parathion: 2-year Chronic Feeding Study of Methyl Parathion in Rats. Monsanto Agricultural Products Company, St. Louis, MO.

TEICHERT, K., SZYMCZYK, T., CONSOLO, S., et al. (1976). "Effect of acute and chronic treatment with dichlorvos on rat brain cholinergic parameters." J. Toxicol. Appl. Pharmacol. 35(1):77-81.

THORPE, E., WILSON, A.B., DIX, K.M, et al. (1972). "Teratological studies with dichlorvos vapor in rabbits and rats." Arch. Toxicol. 30(1):29-38.

THYSSEN, J.T. (1978). Acute Inhalation Toxicity. Report No.7827. Bayer AG, Institute of Toxicology, Wuppertal-Elberfeld, Germany.

THYSSEN, J.T. (1980). Subacute Inhalation Study on Rats. Report No. 9065. Bayer AG, Institute of Toxicology, Wuppertal-Elberfeld, Germany.

TREON, J.F., CLEVELAND, F.P., and CAPPEL, J. (1955). "Toxicity of endrin for laboratory animals." Agric. Food Chem. 3:842-848. 
UCHIDA, Y., NAKATSUKA, H., UKAI, H., et al. (1993). "Symptoms and signs in workers exposed predominantly to xylenes." Int. Arch. Occup. Environ. Health 64:597-605.

WEEKS, M.H., ANGERHOFER, R.A., BISHOP, R., et al. (1979). "The toxicity of hexachloroethane in laboratory animals." Am. Ind. Hyg. Assoc. J. 40:187-199.

WINNEKE, G. (1974). "Behavioral effects of methylene chloride and carbon monoxide as assessed by sensory and psychomotor performance." In: Behavioral Toxicology. (C. Xintaras, B.L. Johnson, I. de Groot, eds.) U.S. Government Printing Office. Washington, DC. pp. 130-144. 